\title{
Tissue Isn't the Issue
}

The approach to clinical conundrums by an expert clinician is revealed through the presentation of an actual patient's case in an approach typical of a morning report. Similar to patient care, sequential pieces of information are provided to the clinician, who is unfamiliar with the case. The focus is on the thought processes of both the clinical team caring for the patient and the discussant.

This icon represents the patient's case. Each paragraph that follows represents the discussant's thoughts.

Lekshmi Santhosh, MD1', Laura Koth, MD1', Thomas E. Baudendistel, MD², Bradley Sharpe, MD¹, Bradley Monash, MD 1,3,*

${ }^{1}$ Department of Medicine, University of California, San Francisco, San Francisco, California; ${ }^{2}$ Kaiser Permanente Medical Center, Oakland, California; ${ }^{3}$ Department of Pediatrics, University of California, San Francisco, San Francisco, California.

A 43-year-old man with a history of asplenia, hepatitis $C$, and nephrolithiasis reported right-flank pain. He described severe, sharp pain that came in waves and radiated to the right groin, associated with nausea and nonbloody emesis. He noted "pink urine" but no dysuria. He had 4prior similar episodes during which he had passed kidney stones, although stone analysis had never been performed. He denied having fevers or chills.

The patient had been involved in a remote motor vehicle accident complicated by splenic laceration, for which he underwent splenectomy. He was appropriately immunized. The patient also suffered from bipolar affective disorder and untreated chronic hepatitis $C$ infection with no evidence of cirrhosis. He smoked one pack of tobacco per day for the last 10 years and reported distant alcohol and methamphetamine use.

Right-flank pain can arise from conditions affecting the lower thorax (effusion, pneumonia, pulmonary embolism), abdomen (hepatobiliary or intestinal disease), retroperitoneum (hemorrhage or infection), musculoskeletal system, peripheral nerves (herpes zoster), or the genitourinary system (pyelonephritis). Pain radiating to the groin, discolored urine (suggesting hematuria), and history of kidney stones increase the likelihood of renal colic from nephrolithiasis.

Less commonly, flank pain and hematuria may present as initial symptoms of renal cell carcinoma, renal infarction, or aortic dissection. The patient's immunosuppression from asplenia and active injection drug use could predispose him to septic emboli to his kidneys. Prior trauma causing aortic injury could predispose himto subsequent dissection.

\footnotetext{
*Address for correspondence: Bradley Monash, MD, University of California, San Francisco, 505 Parnassus Avenue, San Francisco, CA 94143; Email: Bradley. Monash@ucsf.edu
}

Published online first April 25, 2018.

Received: November 8, 2017; Revised: January 1, 2018;

Accepted: February 1, 2018

() 2018 Society of Hospital Medicine DOI 10.12788/jhm.2963
The patient appeared well with a heart rate of 100 beats per minute, blood pressure $122 / 76 \mathrm{mmHg}$, temperature $36.8^{\circ} \mathrm{C}$, respiratory rate 16 breaths per minute, and oxygen saturation $96 \%$ on room air. His cardiopulmonary and abdominal examinations were normal, and he had no costovertebral angle tenderness. His skin was warm and dry without rashes. His white blood cell (WBC) count was $26,000 / \mu \mathrm{L}$; absolute neutrophil count was $22,000 / \mu \mathrm{L}$. Serum chemistries were normal, including creatinine 0.63 $\mathrm{mg} / \mathrm{dL}$, calcium $8.8 \mathrm{mg} / \mathrm{dL}$, and phosphorus $3.1 \mathrm{mg} / \mathrm{dL}$. Lactate was $0.8 \mathrm{mmol} / \mathrm{L}$ (reference range: $0-2.0 \mathrm{mmol} / \mathrm{L}$ ). Urinalysis revealed large ketones, $>50$ red blood cells (RBC) per high power field (HPF), $<5$ WBC per HPF, 1+ calcium oxalate crystals and $\mathrm{pH}$ 6.0. A bedside ultrasound showed mild right hydronephrosis. Computed tomography (CT) with intravenous contrast of his abdomen and pelvis demonstrated diffuse, mildly prominent subcentimeter mesenteric lymphadenopathy and no kidney stones. He was treated with intravenous fluids and pain control, and was discharged with a presumptive diagnosis of a passed kidney stone.

A passed stone would not explain this degree of leukocytosis. The CT results reduce the likelihood of a renal neoplasm, renal infarction, or pyelonephritis. Mesenteric lymphadenopathy is nonspecific, but it may signal underlying infection or malignancy with spread to lymph nodes, or it may be part of a systemic disorder causing generalized lymphadenopathy. Malignant causes of mesenteric lymphadenopathy (with no apparent primary tumor) include testicular cancer, lymphoma, and primary urogenital neoplasms.

His flank pain resolved over the next few days. One week later, he presented with fevers, diffuse headache, painful oral ulcers, pain in the knees and ankles, and a rash involving the face, trunk, and extremities. He was febrile to $38.1^{\circ} \mathrm{C}$, normotensive, with an oxygen saturation of $96 \%$ on room air. He had erythema and swelling of the right eyelid and upper orbit, 2 shallow oral ulcers on the lower buccal mucosa, and bilateral, firm, nontender, $1-\mathrm{cm}$ cervical lymphadenopathy. His visual acuity was normal. His 


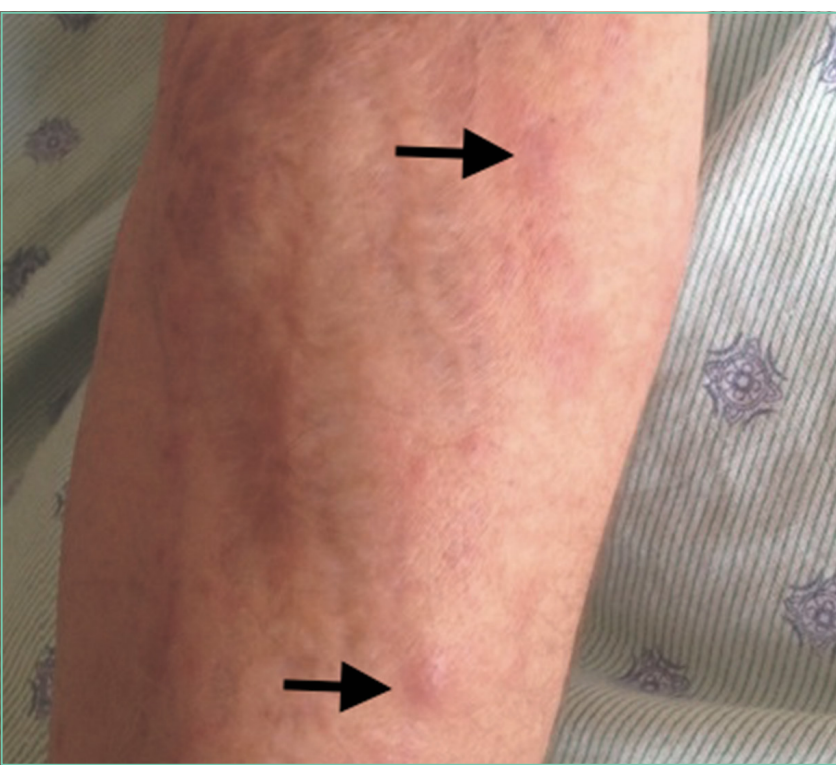

FIG 1. Erythematous papules and plaques

bilateral ankles and knees were warm and tender with small effusions but preserved range of motion. He had innumerable scattered erythematous papules with rare pustules, interspersed with large, erythematous plaques on his face, extremities, back, and buttocks with a predilection for previous scars and tattoos (Figure 1). He also had tender, erythematous nodules on his anterior lower extremities. His neurological exam was normal.

The lower extremity nodules are consistent with erythema nodosum, which may be observed in numerous infectious and noninfectious illnesses. The rapid tempo of this febrile illness mandates early consideration of infection. Splenectomized patients are at risk for overwhelming post-splenectomy infection from encapsulated organisms, although this risk is significantly mitigated with appropriate immunization. The patient is at risk of bacterial endocarditis, which could explain his fevers and polyarthritis, although plaques, pustules, and oral ulcers would be unusual. Disseminated gonococcal infection causes fevers, oral lesions, polyarthritis and pustular skin lesions, but plaques are uncommon. Disseminated mycobacterial and fungal infections may cause oral ulcers, but affected patients tend to be severely ill and have profound immunosuppression. Secondary syphilis may account for many of the findings; however, oral ulcers would be unusual, and the rash tends to be more widespread, with a predilection for the palms and soles. Human immunodeficiency virus (HIV) can cause oral ulcers and is the chief viral etiology to consider.

Noninfectious illnesses to consider include neoplasms and connective tissue diseases. Malignancy would be unlikely to manifest this abruptly or produce a paraneoplastic disorder with these features. Among the connective tissue diseases, sarcoidosis warrants consideration in this patient with adenopathy, erythema nodosum, arthritis, and a predilection for skin changes in prior scars. However, it is uncommon for sarcoidosis to present so explosively. Painful oral and genital ulcers, pustular rash, polyarthritis, and erythema nodosum occur in Behçet's disease, which is associated with pathergy (an exaggerated cutaneous response to minor trauma). Patients with Behçet's may have eye involvement, including uveitis and a hypopion, and may develop vascular aneurysms in the pulmonary, intracranial, or visceral arteries. Renal artery involvement could cause hematuria and flank pain.

The patient described severe fatigue and drenching night sweats for two months prior to admission. He denied dyspnea or cough. He was born in the southwestern United States and had lived in California for almost a decade. He had been incarcerated for a few years and released three years prior. He had intermittently lived in homeless shelters, but currently lived alone in downtown San Francisco. He had traveled remotely to the Caribbean, and more recently traveled frequently to the Central Valley in California. The patient formerly worked as a pipe-fitter and welder. He denied animal exposure or recent sick contacts. He was sexually active with women, and intermittently used barrier protection.

His years in the southwestern United States may have exposed the patient to blastomycosis or histoplasmosis; both can mimic mycobacterial disease. Blastomycosis demonstrates a slightly stronger predilection for spreading to the bones, genitourinary tract, and central nervous system, whereas histoplasmosis is a more frequent cause of polyarthrtitis and mesenteric adenopathy. The patient's travel to the Central Valley, California raises the possibility of coccidioidomycosis, which typically starts with pulmonary disease prior to dissemination to bones, skin, and other less common sites. Pipe-fitters are predisposed to asbestos-related illnesses, including lung cancer and mesothelioma, which would not explain this patient's presentation. Incarceration and high-risk sexual practices increase his risk for tuberculosis, HIV, and syphilis. Widespread skin involvement is more characteristic of syphilis or primary HIV infection than of disseminated fungal or mycobacterial infection.

WBC measured 29,000/uL with a neutrophilic predominance. His peripheral blood smear was unremarkable. A comprehensive metabolic panel was normal. Lactate dehydrogenase (LDH) was $317 \mathrm{U} / \mathrm{L}$ (reference range 140-280 $\mathrm{U} / \mathrm{L}$ ). Erythrocyte sedimentation rate (ESR) was $39 \mathrm{~mm} / \mathrm{hr}$ (reference range $<20 \mathrm{~mm} / \mathrm{hr}$ ) and C-reactive protein (CRP) was $66 \mathrm{mg} / \mathrm{L}$ (reference range $<6.3 \mathrm{mg} / \mathrm{L}$ ). Blood, urine, and throat cultures were sent. Chest radiograph showed clear lungs without adenopathy. Ankle and knee radiographs identified small effusions bilaterally without bony abnormalities. CT of his brain showed a small, hypodense lesion in the right lacrimal gland. A lumbar puncture with cerebrospinal fluid (CSF) analysis showed absence of RBCs; WBC, $2 / \mu \mathrm{L}$; protein, $35 \mathrm{mg} / \mathrm{dL}$; glucose, $62 \mathrm{mg} / \mathrm{dL}$; negative gram stain. CSF bacterial and fungal cultures, venereal disease research laboratory (VDRL), herpes simplex virus 
polymerase chain reaction (HSV PCR), and cryptococcal antigen were sent for laboratory analysis. The patient was started on vancomycin and aztreonam.

Lesions of the lacrimal gland feature multiple causes, including autoimmune diseases (Sjögren's, Behçet's disease), granulomatous diseases (sarcoidosis, granulomatosis with polyangiitis), neoplasms (salivary gland tumors, lymphoma), and infections. Initiating broad-spectrum antibiotics is reasonable while awaiting additional information from blood and urine cultures, serologies for HIV and syphilis, and purified protein derivative or interferon-gamma release assay (IGRA).

If these tests fail to reveal a diagnosis, the search for atypical infections and noninfectious possibilities should expand. Histoplasmosis and blastomycosis would be the most likely fungal diseases to account for his arthritis and adenopathy. Coccidioidomycosis is less likely in light of the normal chest radiograph. Computed tomography of the chest would be reasonable to look for adenopathy, which would strengthen the case for lymphoma or sarcoidosis, and may also identify a potential site to biopsy to establish these diagnoses.

The patient continued to have intermittent fevers, sweats, and malaise over the next 3 days. All bacterial and fungal cultures remained negative, and antibiotics were discontinued. Rheumatoid factor, anticyclic citrullinated peptide, antinuclear antibody, and cryoglobulins were negative. Serum C3, C4, and angiotensin-converting enzyme (ACE) levels were normal. $A$ rapid plasma reagin (RPR), HIV antibody, IGRA, and serum antibodies for Coccidioides, histoplasmosis, and West Nile virus were negative. Urine nucleic acid amplification testing for gonorrhea and chlamydia was negative. CSF VDRL, HSV PCR and cryptococcal antigen were negative. HSV culture from an oral ulcer showed no growth. The patient had a reactive hepatitis $C$ antibody with a viral load of 3 million virus equivalents $/ \mathrm{mL}$.

The additional test results lower the likelihood of an acute infection. Uncontrolled hepatitis C increases the risk of several noninfectious manifestations. The normal results for serum complements and cryoglobulins effectively rule out cryoglobulinemia. Patients with hepatitis $C$ have an increased risk of lymphoma, which could account for the subacute fevers, night sweats, adenopathy, elevated LDH, and the right orbital mass, but less likely for the oral ulcers, arthritis, and skin manifestations. Sarcoidosis is less likely given the lack of hilar adenopathy, relatively abrupt onset of multisystem disease, and the presence of oral ulcers. Behçet's disease could account for his oral ulcers, erythema nodosum, and distribution of papules, pustules, and plaques with the predilection for scars and tattoos. Behçet's could also explain the arthritis, the hematuria if the patient had renal artery involvement, and the orbital lesion. However, lymphadenopathy is not a prominent feature. At this point, tissue should be obtained for histopathology (to assess for vasculitis or granulomatous infiltration) and flow cytometry.

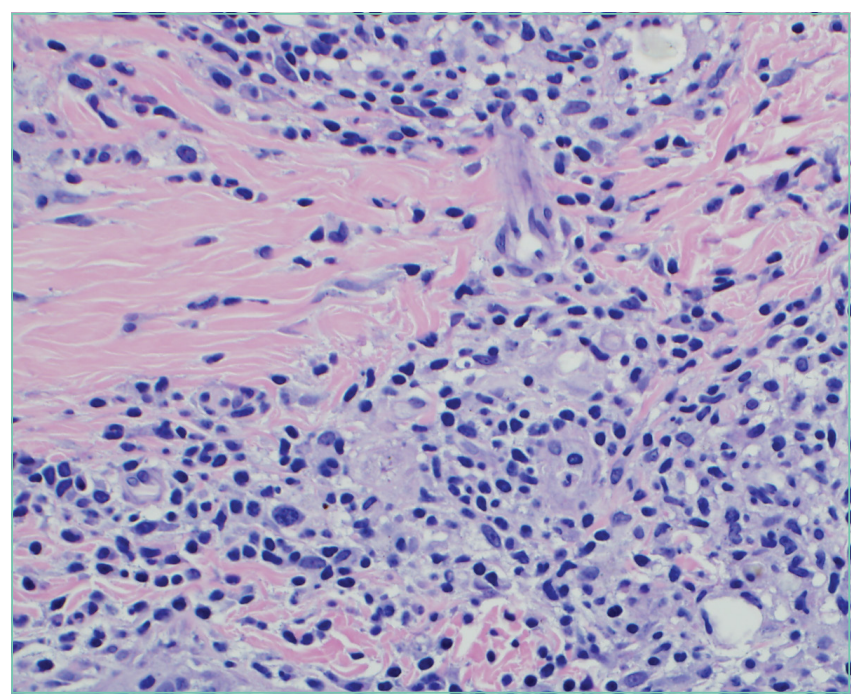

FIG 2. Inflammation at the interface of the dermis and the subcutis with neutrophils, histiocytes and fatty microcysts.

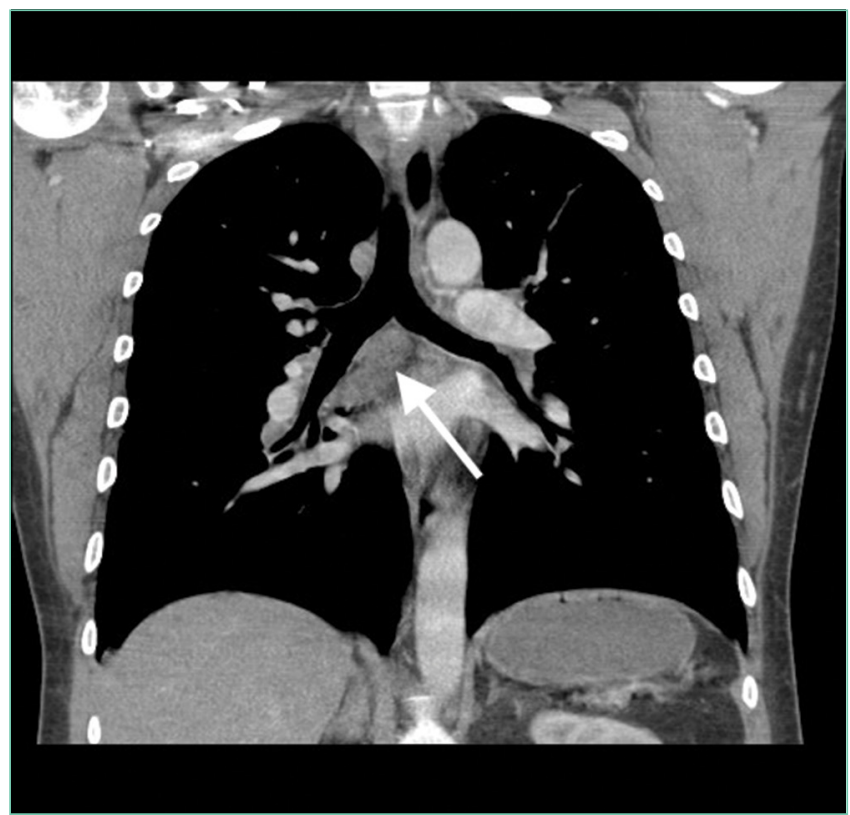

FIG 3. Noncontrast chest computed tomography (CT) showing subcarinal lymphadenopathy (arrow)

Biopsies of the skin plaques associated with old scars revealed granulomatous infiltrates. Fine-needle aspiration (FNA) of a submental lymph node showed benign lymphoid tissue; flow cytometry was negative for malignancy. Punch biopsy of the right anterior thigh nodule demonstrated superficial and deep perivascular infiltrate of lymphocytes in the dermis and superficial subcutis, and inflammation at the interface of the dermis and the subcutis with neutrophils, histiocytes, and fatty microcysts (Figure 2). All biopsies stained negative for fungi and mycobacteria. High-resolution CT scan of the chest demonstrated increased number and size of multiple lymph nodes of the mediastinum, hila, and upper abdomen (Figure 3). 
Biopsy results and flow cytometry substantially lower the probability of lymphoma. The presence of granulomas on skin biopsy and the extensive lymphadenopathy are not characteristic of Behçet's. Biopsy from the leg describes erythema nodosum

The most likely diagnosis is Löfgren's syndrome, a variant of sarcoidosis characterized by erythema nodosum, bilateral hilar lymphadenopathy, and polyarthralgias or polyarthritis. Löfgren's syndrome may include fevers, uveitis, widespread skin lesions and other systemic manifestations. Sarcoidosis could explain the lacrimal gland lesion, and could manifest with recurrent kidney stones. Oral lesions may occur in sarcoidosis. A normal serum ACE level may be observed in up to half of patients. The lack of visualized granulomas on the submental node FNA may reflect sampling error, lower likelihood of visualizing granulomas on FNA (compared with excisional biopsy), or biopsy location (hilar nodes are more likely to demonstrate sarcoid granulomas).

Although Löfgren's syndrome is often self-limited, treatment can ameliorate symptoms. Nonsteroidal anti-inflammatory medication can be tried first, with prednisone reserved for refractory cases.

The constellation of bilateral hilar adenopathy, arthritis, and erythema nodosum was consistent with Löfgren's syndrome, further supported by granulomatous infiltrates on biopsy. The patient's symptoms resolved with naproxen. He was scheduled for follow-up in dermatology and rheumatology clinics and was referred to hepatology for management of hepatitis $C$.

\section{COMMENTARY}

Sarcoidosis is a multisystem granulomatous disease of unclear etiology. The disease derives its name from Boeck's 1899 report describing benign cutaneous lesions that resembled sarcomas. ' Sarcoidosis most commonly manifests as bilateral hilar adenopathy and pulmonary infiltrates, but may impact any tissue or organ, including the eyes, nonhilar lymph nodes, liver, spleen, joints, mucous membranes, and skin. Nephrolithiasis may result from hypercalcemia and/or hypercalciuria (related to granulomatous production of 1,25 vitamin D) and can be the presenting feature of sarcoidosis. ${ }^{2}$ Less common presentations include neurologic sarcoidosis (which can present with seizures, aseptic meningitis, encephalopathy, neuroendocrine dysfunction, myelopathy and peripheral neuropathies), cardiac sarcoidosis (which may present with arrhythmias, valvular dysfunction, heart failure, ischemia, or pericardial disease), and Heerfordt syndrome (the constellation of parotid gland enlargement, facial palsy, anterior uveitis, and fever). Sarcoidosis may mimic other diseases, including malignancy, idiopathic pulmonary fibrosis, and infiltrative tuberculosis. ${ }^{3}$ Sarcoidosis-like reactions have occurred in response to malignancy and medications. ${ }^{4}$

The patient's rash demonstrated a predilection for areas of prior scarring, which has a limited differential diagnosis. Keloids and hypertrophic scars occur at sites of former surgical wounds, lacerations, or areas of inflammation. Pruritic urticarial papules and plaques of pregnancy (PUPPP) is a benign inflammatory condition where papules cluster in areas of prior striae. Cutaneous lesions of Behçet's syndrome display pathergy, where pustular response is observed at sites of injury. Granulomatous infiltration in sarcoidosis may demonstrate a predilection for scars and tattoos (ie, scar or tattoo sarcoidosis). ${ }^{5}$ Sarcoidosis can have other cutaneous manifestations, including psoriaform, ulcerative, or erythrodermic lesions; subcutaneous nodules; scarring or nonscarring alopecia; and lupus pernio - violaceous, nodular and plaque-like lesions on the nose, earlobes, cheeks, and digits. ${ }^{5}$

Löfgren's syndrome is a distinct variant of sarcoidosis. In 1952, Dr. Löfgren described a case series of patients with bilateral hilar lymphadenopathy and coexisting erythema nodosum and polyarthralgia. ${ }^{6}$ The epidemiology favors young women. 'Patients with Löfgren's syndrome present acutely (as in this case), which differs from the typical subacute course observed with sarcoidosis. In addition to the classic presentation described above, patients with Löfgren's syndrome may demonstrate additional manifestations of sarcoidosis, including fevers, peripheral adenopathy, arthritis, and granulomatous skin lesions. Painful symptoms may require short-term anti-inflammatory treatments. Most patients do not require systemic immunosuppression. Symptoms usually decrease over several months, and the majority of patients experience complete remission within years. Rare recurrences have been described up to several years. ${ }^{8}$

In confirming the diagnosis of sarcoidosis, current guidelines recommend exclusion of other diseases that present similarly, a work-up that generally includes compatible laboratory tests and imaging, and histologic demonstration of noncaseating granulomas. ${ }^{9}$ However, Löfgren's syndrome is a notable exception. The constellation of fever, bilateral hilar adenopathy, polyarthralgia, and erythema nodosum suffices to diagnose Löfgren's syndrome as long as the disease remits rapidly and spontaneously. ${ }^{9}$ Thus, in this case, although granulomatous infiltrates were confirmed on biopsy, the diagnosis of Löfgren's syndrome could have been based on clinical and radiologic features alone.

\section{KEY LEARNING POINTS}

- Sarcoidosis is a multisystem granulomatous disease that most commonly presents with bilateral hilar adenopathy and pulmonary infiltrates but can also present atypically, including with nephrolithiasis from hypercalcemia, neurologic syndromes, and cardiac involvement.

- Löfgren's syndrome, a variant of sarcoidosis, is characterized by relatively acute onset of fevers, erythema nodosum, bilateral hilar adenopathy, and polyarthralgia or polyarthritis. Most patients recover and manifest complete remission.

- A limited differential exists for rashes with a predilection for areas of tattoos and prior scarring, including keloids, PUPPP, Behçet's disease, and granulomatous infiltration.

Disclosure: There are no conflicts of interest or financial disclosures to report. 


\section{References}

1. Multiple Benign Sarcoids of the Skin. JAMA. 1899;XXXIII(26):1620-1621.

2. Rizzato G, Fraioli P, Montemurro L. Nephrolithiasis as a presenting feature of chronic sarcoidosis. Thorax. 1995;50(5):555-559.

3. Romanov V. Atypical variants of clinical course of sarcoidosis. Eur Respir J. 2014;44(58):3782.

4. Arish N, Kuint R, Sapir E, et al. Characteristics of Sarcoidosis in Patients with Previous Malignancy: Causality or Coincidence? Respiration. 2017;93(4): 247-252.

5. Marchell RM, Judson MA. Chronic cutaneous lesions of sarcoidosis.
Clin Dermatol. 2007;25(3):295-302.

6. Löfgren S. The Bilateral Hilar Lymphoma Syndrome. Acta Med Scand. 1952;142(4):265-273.

7. Mañá J, Gómez-Vaquero C, Montero A et al. Löfgren's syndrome revisited: a study of 186 patients. Am J Med. 1999;107(3):240-245.

8. Gran J, Bohmer E. Acute Sarcoid Arthritis: A Favourable Outcome? Scand J Rheumatol. 1996;25(2):70-73.

9. American Thoracic Society. Statement on Sarcoidosis. Am J Respir Crit Care Med. 1999;160:736-755. 